\title{
Differences in Left Ventricular Global Function and Mechanics in Paralympic Athletes with Cervical and Thoracic Spinal Cord Injuries
}

\author{
Katharine D. Currie ${ }^{1}$, Christopher R. West ${ }^{1,2}$ and Andrei V. Krassioukov ${ }^{1,3,4 *}$ \\ ${ }^{1}$ Autonomic Research Laboratory, International Collaboration on Repair Discoveries, Faculty of Medicine, University of British \\ Columbia, Vancouver, BC, Canada, ${ }^{2}$ Translational Integrative Physiology Laboratory, Faculty of Education, School of \\ Kinesiology, University of British Columbia, Vancouver, BC, Canada, ${ }^{3}$ Division of Physical Medicine and Rehabilitation, \\ Faculty of Medicine, University of British Columbia, Vancouver, BC, Canada, ${ }^{4}$ GF Strong Rehabilitation Centre, Vancouver \\ Coastal Health, Vancouver, BC, Canada
}

\section{OPEN ACCESS}

Edited by: Gregoire P. Millet, University of Lausanne, Switzerland

Reviewed by: Pierre-Marie Leprêtre, Université de Picardie Jules Verne, France

Stefanos Volianitis, Aalborg University, Denmark

*Correspondence: Andrei V. Krassioukov

krassioukov@icord.org

Specialty section: This article was submitted to

Exercise Physiology, a section of the journal

Frontiers in Physiology

Received: 11 September 2015 Accepted: 07 March 2016

Published: 29 March 2016

Citation:

Currie KD, West CR and Krassioukov AV (2016) Differences in Left Ventricular Global Function and Mechanics in Paralympic Athletes with Cervical and Thoracic Spinal Cord Injuries. Front. Physiol. 7:110 doi: 10.3389/fphys.2016.00110
Following a spinal cord injury, there are changes in resting stroke volume (SV) and its response to exercise. The purpose of the following study was to characterize resting left ventricular structure, function, and mechanics in Paralympic athletes with tetraplegia (TETRA) and paraplegia (PARA) in an attempt to understand whether the alterations in SV are attributable to inherent dysfunction in the left ventricle. This retrospective study compared Paralympic athletes with a traumatic, chronic ( $>1$ year post-injury), motor-complete spinal cord injury (American Spinal Injury Association Impairment Scale A-B). Eight male TETRA wheelchair rugby players (34 \pm 5 years, C5-C7) and eight male PARA alpine skiers (35 \pm 5 years, T4-L3) were included in the study. Echocardiography was performed in the left lateral decubitus position and indices of left ventricular structure, global diastolic and systolic function, and mechanics were derived from the average across three cardiac cycles. Blood pressure was measured in the supine and seated positions. All results are presented as TETRA vs. PARA. There was no difference in left ventricular dimensions between TETRA and PARA. Additionally, indices of global diastolic function were similar between groups including isovolumetric relaxation time, early $(E)$ and late $(A)$ transmitral filling velocities and their ratio $(E / A)$. While ejection fraction was similar between TETRA and PARA ( $59 \pm 4 \%$ vs. $61 \pm 7 \%, p=0.394$ ), there was evidence of reduced global systolic function in TETRA including lower SV (62 $\pm 9 \mathrm{ml}$ vs. $71 \pm 6 \mathrm{ml}$, $p=0.016)$ and cardiac output ( $3.5 \pm 0.6 \mathrm{~L} / \mathrm{min}$ vs. $5.0 \pm 0.9 \mathrm{~L} / \mathrm{min}, p=0.002)$. Despite this observation, several indices of systolic and diastolic mechanics were maintained in TETRA but attenuted in PARA including circumferential strain at the level of the papillary muscle ( $-23 \pm 4 \%$ vs. $-15 \pm 6 \%, p=0.010)$ and apex $(-36 \pm 10 \%$ vs. $-23 \pm 5 \%$, $p=0.010$ ) and their corresponding diastolic strain rates (papillary: $1.90 \pm 0.63 \mathrm{~s}^{-1} \mathrm{vs}$. $1.20 \pm 0.51 s^{-1}, p=0.028$; apex: $3.03 \pm 0.71 s^{-1}$ vs. $1.99 \pm 0.69 s^{-1}, p=0.009$ ). All blood pressures were lower in TETRA. The absence of an association between reduced global systolic function and mechanical dysfunction in either TETRA or PARA suggests any reductions in SV are likely attributed to impaired loading rather than inherent left ventricular dysfunction.

Keywords: athletes, echocardiography, paraplegia, strain, stroke volume, tetraplegia 


\section{INTRODUCTION}

The classification of Paralympic athletes is sports specific and considers physical, visual and/or intellectual impairments. Typically athletes with a spinal cord injury (SCI) are competing against individuals with a similar lesion level (i.e., cervical or thoracic SCI). However, there are sporting competitions where individuals with cervical and thoracic SCI are competing against each other, in addition to other non-SCI athletes. The consideration of how the lesion level may influence exercise performance is therefore necessary to ensuring fair competition between the diverse population of Paralympic athletes.

One contributing factor to exercise performance is the capacity of the cardiovascular system to adequately respond to the body's demands. This includes the responses of the heart, vasculature, and adrenal medulla. Following SCI, there are lesion level dependent alterations in how the cardiovascular system responds to an exercise stimulus. While sympathetic preganglionic neurons originate in the thoracic and upper lumbar segments of the spinal cord (T1-L2), sympathetic innervation of target organs is segmental. Innervation of the heart and vascular smooth muscle of the upper body arise from the high thoracic segments (T1-T5), while innervation of the vascular smooth muscle of the lower body and adrenal medulla arise from the lower thoracic and lumbar segments (T6-L2). Cervical SCI results in the most dramatic alterations in cardiovascular outcomes at rest and in response to exercise, due to the disruption of descending sympathetic input to target organs below the level of injury (Krassioukov, 2009). We recently confirmed this by demonstrating individuals with tetraplegia who had an autonomic complete SCI also reached attenuated peak heart rates of $102 \pm 34 \mathrm{bpm}$ while individuals with tetraplegia and autonomic incomplete SCI reached higher peak heart rates $(161 \pm 20 \mathrm{bpm})$ (Currie et al., 2015). Additional cardiovascular responses that are attenuated during exercise in individuals with tetraplegia include the changes in blood pressure (Thijssen et al., 2009), catecholamine levels (Schmid et al., 1998a,b), and stroke volume (SV) (Kessler et al., 1986; Hostettler et al., 2012). Contrary to tetraplegia, individuals with high and low paraplegia have either partial or full preservation of descending sympathetic pathways to the heart, vascular smooth muscle and adrenal medulla, and therefore are capable of mounting an appropriate cardiovascular response during exercise. Previous research has demonstrated individuals with paraplegia are capable of reaching peak heart rates in range of their age-predicted heart rate maximum (Hopman et al., 1992, 1993; Jacobs et al., 2002) and display exercise-induced increases in circulating catecholamines (Schmid et al., 1998a,b) and blood pressure (Dela et al., 2003; Claydon et al., 2006). SV responses to aerobic exercise in individuals with paraplegia, however, are inconclusive with previous research either demonstrating no change (Davis and Shephard, 1988; Hopman et al., 1993; Raymond et al., 2001; Theisen et al., 2001) or small increases (Davis et al., 1987; Hopman et al., 1992; Raymond et al., 1999). Overall, exerciseinduced increases in cardiac output $(\dot{Q})$ in individuals with SCI are primarily driven by changes in heart rate, with individuals with paraplegia exhibiting a greater capacity to increase $\dot{Q}$ during exercise.

Altered SV responses in SCI have primarily been attributed to a reduction in loading (i.e., decreased venous return) (Theisen, 2012). However, no studies have examined whether factors inherent to the left ventricle (LV) per se are also a contributing factor. Echocardiographic assessments of LV mechanics are a non-invasive tool that may help to determine if changes in LV performance following SCI are associated with SV reductions. Indices of LV mechanics include strain $(\varepsilon)$ and strain rates (SR) which assess the degree and rate of myocardial deformation in longitudinal, radial and circumferential axes; rotation (Rot) and rotation rates (RotR) of the base and apex of the LV; and overall LV twist and twisting/untwisting rates (Voigt et al., 2015). In clinical conditions, such as chronic heart failure, there is a concomitant reduction in global LV systolic function [SV; LV ejection fraction (EF)] and LV systolic mechanics (Leung and Ng, 2010; Ma et al., 2014), and recent evidence suggests lower $\varepsilon$ in these patients is associated with a reduced exercise capacity (Hasselberg et al., 2015). Additionally, indices of LV mechanics are sensitive to increases and decreases in sympathetic activity (Weidemann et al., 2002; Akagawa et al., 2007), which may provide insight into the influence of lesion level on LV performance. Few studies have performed echocardiographic assessments in athletes with SCI (Huonker et al., 1998; Gates et al., 2002; Schumacher et al., 2009; Maggioni et al., 2012; West et al., 2012a; De Rossi et al., 2014), with none of these investigations examining LV mechanics. Given evidence that global LV systolic dysfunction is associated with impairments in LV mechanics, and that these functional changes may limit exercise performance, the examination of LV structure, function and mechanics in athletes with SCI may help to elucidate potential factors limiting cardiac responses to exercise. Therefore, the purpose of this study was to compare resting LV structure, function and mechanics in high-performance athletes with SCI.

\section{MATERIALS AND METHODS}

\section{Participants}

This was a retrospective comparison of echocardiography data collected at both summer and winter Paralympic events. Inclusion criteria included males between 18-60 years of age who had sustained a chronic ( $>1$ year post-injury), motorcomplete [American Spinal Injury Association Impairment Scale (AIS) A-B], traumatic SCI, and who have been competing at the international level for at least 3 years. Eight Paralympic wheelchair rugby players with a cervical SCI (TETRA) and eight Paralympic alpine skiers with a thoracic SCI (PARA) met the criteria and were included in the study. The neurological level of injury and AIS was confirmed using the International Standards for Neurological Classification of SCI (Kirshblum et al., 2011). Exclusion criteria for the study included any history of cardiovascular disease or acute illness/infection, which was confirmed with a verbal medical history, and any language or cognitive barrier that prevented the participant from following English instructions. The International Paralympics Committee, University of British Columbia Clinical Research Ethics Board, 
and Brunel University Research Ethics Board approved all protocols, which conformed to the Declaration of Helsinki, and all individuals provided written informed consent prior to participation. Prior to testing, participants were instructed to abstain from food and drink for $4 \mathrm{~h}$, alcohol and caffeine for $12 \mathrm{~h}$, and exercise for $24 \mathrm{~h}$. On the day of testing, all participants were instructed to void their bladder to reduce the potential influence of sympathetic reflex activation on blood pressure.

\section{Blood Pressure Assessments}

Blood pressures were measured from the left brachial artery using an automated machine (Dinamap Pro 300 V2; GE Healthcare, Milwaukee, USA). Seated and supine measurements were collected in duplicate following 5 and $10 \mathrm{~min}$ of quiet rest, respectively.

\section{Assessments}

Cardiac images were collected with the participants in the left lateral decubitus position using two-dimensional echocardiography (Vivid 7; GE Healthcare, Horton, Norway) according to the recommendations of the American Society for Echocardiography (Quiñones et al., 2002; Lang et al., 2005; Voigt et al., 2015). Heart rate was measured continuously using a single-lead electrocardiogram. Images were analyzed offline using dedicated software (EchoPAC; GE Healthcare, Horten, Norway) by a single blinded investigator, and the average of three cardiac cycles is presented.

Indices of LV structure were measured at end-diastole (d) and end-systole (s) from the parasternal long axis view, including LV internal diameter (LVID), and interventricular septal wall (SWT), and posterior wall (PWT) thickness. Relative wall thickness $(\mathrm{RWT})$ was calculated as $[(2 \times \mathrm{PWTd}) / \mathrm{LVIDd}]$. LV mass (LVMI) was calculated according to an established formula (Devereux et al., 1986), and indexed to body surface area (Du Bois and Du Bois, 1989). Modified single-plane Simpson's method was used to analyze apical four-chamber views to determine end-diastolic (EDV) and end-systolic (ESV) volumes, and the global systolic function outcomes of SV, EF, and $\dot{Q}$ which was calculated as the product of SV and heart rate. Global diastolic function outcomes were determined from pulsed-wave Doppler at the tips of the mitral valve leaflet. Outcomes included early (E) and late (A) transmitral filling velocities and their ratio (E/A), and isovolumetric relaxation time (IVRT).

Indices of LV mechanics were derived from 2D speckletracking analysis of apical four-chamber and parasternal short axis images at the level of the mitral valve (basal), papillary muscle (mid), and apex (apical) using established guidelines (Mor-Avi et al., 2011). Using a cubic spline algorithm, raw speckle-tracking traces were interpolated by customized post-processing software (2D Strain Analysis Tool, Stuttgart, Germany) into 600 points in systole and 600 points in diastole to control for heart rate differences. Peak $\varepsilon$ and SR in systole and diastole were determined from short-axis (radial, circumferential) and four-chamber (longitudinal) images. Peak Rot and RotR in systole and diastole were determine at the basal and apical levels, and twist was determined as the maximum value from subtracting frame-by-frame basal and apical Rot data. Peak systolic twisting velocity and early diastolic untwisting velocity were determined the same way using frame-by-frame basal and apical RotR data.

\section{Statistical Analyses}

Statistical analyses were performed using Statistical Package for Social Science software (IBM Corporation, Armonk, NY, USA). Data were assessed for normality using Shapiro-Wilk tests and Q-Q plot analyses. Between group differences were determined using independent $t$-tests and Mann-Whitney $U$-tests for normally and non-normally distributed data, respectively. Effect sizes were calculated using Cohen's $d$. Data are presented as mean $\pm \mathrm{SD}$ unless otherwise noted, with $P<0.05$ considered statistically significant.

\section{RESULTS}

Individual and group values for participant characteristics are presented in Table 1. Body surface area was similar in TETRA $\left(1.84 \pm 0.13 \mathrm{~m}^{2}\right)$ and PARA $\left(1.93 \pm 0.15 \mathrm{~m}^{2}, p=0.232\right)$. Selfreported training history was available in 5 of 8 TETRA and all 8 PARA athletes. There was no significant difference in the number of years competing at an international level (TETRA: $11 \pm 4$ years; PARA: $8 \pm 5$ years, $p=0.275$ ) or weekly training volume (TETRA: $10 \pm 3$ hours/week; PARA: $16 \pm 13$ hours/week, $p=$ $0.192)$. For seated and supine hemodynamics, heart rates were

\section{TABLE 1 | Participant characteristics.}

Lesion Level AIS TPI (yr) Age (yr) Height (m) Mass (kg)

\begin{tabular}{|c|c|c|c|c|c|c|}
\hline \multicolumn{7}{|l|}{ TETRA } \\
\hline 1 & C5 & A & 14 & 38 & 1.68 & 61 \\
\hline 2 & C6 & A & 11 & 38 & 1.76 & 62 \\
\hline 3 & C6 & A & 12 & 37 & 1.76 & 63 \\
\hline 4 & C6 & B & 12 & 25 & 1.76 & 55 \\
\hline 5 & C6 & $\mathrm{B}$ & 20 & 36 & 1.80 & 72 \\
\hline 6 & $\mathrm{C} 7$ & $\mathrm{~B}$ & 6 & 29 & 1.86 & 80 \\
\hline 7 & C7 & A & 15 & 32 & 1.83 & 70 \\
\hline 8 & $\mathrm{C7}$ & $\mathrm{B}$ & 19 & 33 & 1.85 & 71 \\
\hline Mean \pm SD & - & - & $14 \pm 5$ & $34 \pm 5$ & $1.79 \pm 0.06$ & $67 \pm 8$ \\
\hline \multicolumn{7}{|l|}{ PARA } \\
\hline 1 & $\mathrm{~T} 4$ & A & 8 & 39 & 1.96 & 83 \\
\hline 2 & $\mathrm{~T} 4$ & A & 24 & 41 & 1.76 & 72 \\
\hline 3 & T5 & A & 20 & 38 & 1.77 & 70 \\
\hline 4 & T8 & A & 7 & 24 & 1.85 & 64 \\
\hline 5 & $\mathrm{~T} 10$ & A & 14 & 35 & 1.85 & 85 \\
\hline 6 & T10 & A & 19 & 32 & 1.68 & 58 \\
\hline 7 & T11 & A & 11 & 35 & 1.86 & 72 \\
\hline 8 & L3 & A & 18 & 34 & 1.86 & 72 \\
\hline Mean \pm SD & - & - & $15 \pm 6$ & $35 \pm 5$ & $1.82 \pm 0.08$ & $72 \pm 9$ \\
\hline
\end{tabular}

A, motor- and sensory-complete; AIS, American Spinal Injury Association Impairment Scale; B, motor-complete and sensory-incomplete; C, cervical; L, lumbar; T, thoracic; TPl, time post-injury. 

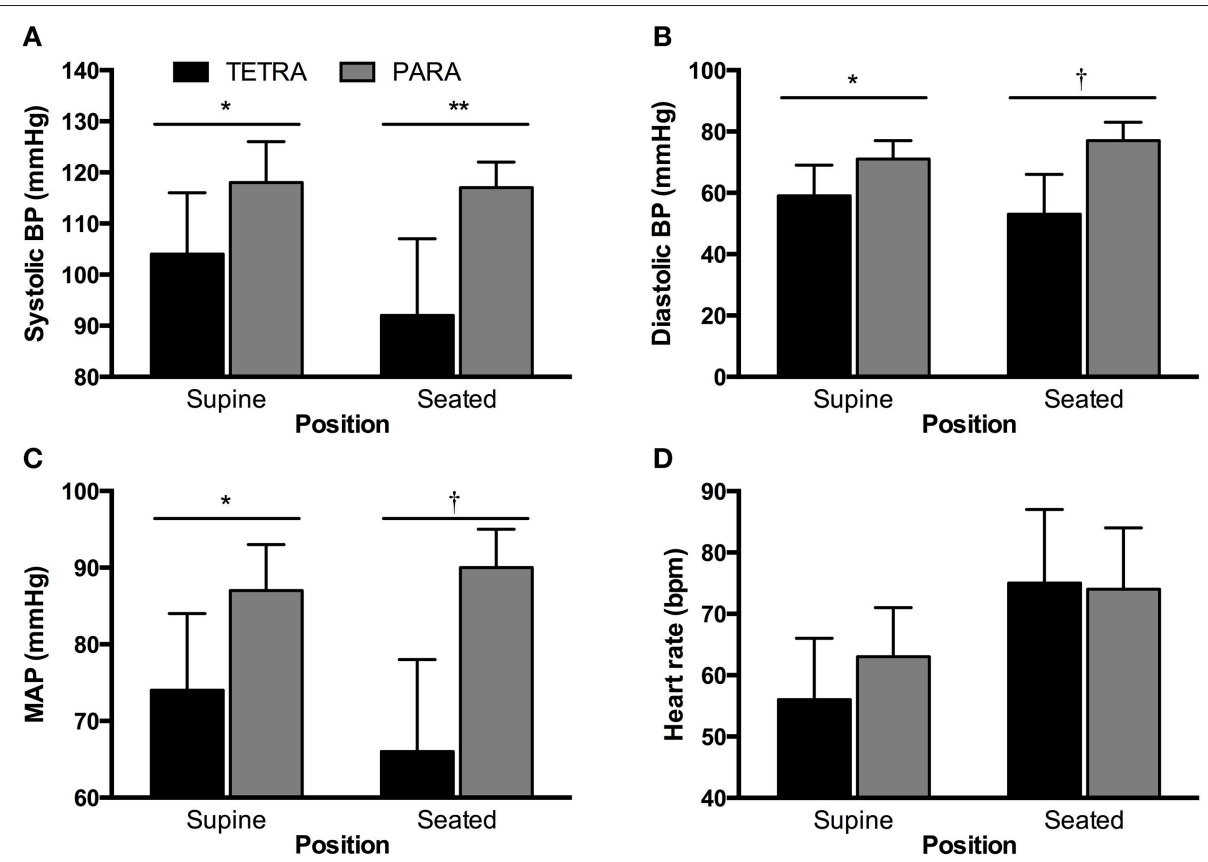

FIGURE 1 | Supine and seated systolic (A) and diastolic (B) blood pressure (BP), mean arterial pressure (C, MAP) and heart rate (D) for TETRA (black bars) and PARA (gray bars) groups. Between-group differences: ${ }^{*} p<0.05 ;{ }^{* *} p<0.01 ;{ }^{\dagger} p \leq 0.001$.

similar between groups while all blood pressure values were lower in TETRA compared to PARA (Figure 1).

Indices of LV structure and global function are presented in Table 2. Structure and global diastolic function was similar between groups, while global systolic function was reduced in TETRA compared to PARA including slower heart rates and smaller SV and $\dot{Q}$. Indices of LV mechanics are presented in Table 3. Basal Rot and RotR in both systole and diastole were higher in TETRA. The TETRA group also presented with higher circumferential $\varepsilon$ (Figure 2) and diastolic SR at the mid and apical levels compared to PARA.

\section{DISCUSSION}

This is the first study to examine the influence of lesion level on resting LV structure, function and mechanics in Paralympic athletes with SCI. We observed no difference in LV dimensions or global diastolic function, while global systolic function was reduced in TETRA compared with PARA. Despite these observations, there were no indicators of LV mechanical dysfunction in the TETRA group, while PARA presented with attenuated systolic and diastolic mechanics. This observation may suggest a training induced improvement in mechanical efficiency in PARA that would increase their capacity to respond during an exercise bout.

The athletes included in this retrospective study were from two distinct sporting events. While we would consider any Paralympic athlete as highly trained, the nature of the training regiments for wheelchair rugby and alpine skiing are unique and incorporate different amounts of endurance and resistance exercise. While there is evidence in the able-bodied literature that endurance and resistance exercise training elicit differential cardiac adaptations (Spence et al., 2011), evidence from a cross-sectional examination of athletes with paraplegia found no differences in LV dimensions or global LV function between power-trained and endurance-trained athletes (Gates et al., 2002). Furthermore, endurance and resistance training in individuals with paraplegia has been shown to elicit similar improvements in peak oxygen uptake (Jacobs, 2009). Thus, it is unlikely that the differences observed between the TETRA and PARA groups are attributed to the differences in training modality. Both groups had similar weekly volumes of exercise training, and had been engaging in international competition for a similar duration. Additionally, the global LV indices observed in PARA athletes are comparable to values recently reported by De Rossi et al. (2014) in their examination of endurance trained athletes with paraplegia (i.e., wheelchair basketball, handball and tennis). While the training regiments for wheelchair rugby and alpine skiing are different, they do incorporate both aerobic and resistance exercises, which based on the literature to date, do not appear to elicit specific cardiac adaptations in individuals with SCI.

Exercise training may exert differential effects on global LV function depending on lesion level. De Rossi et al. (2014) demonstrated increased global diastolic function in athletes with tetraplegia and paraplegia relative to their sedentary peers with tetraplegia and paraplegia. However, SV was reduced in both sedentary and athletic groups with tetraplegia and only improved in athletes with paraplegia relative to sedentary individuals with paraplegia. Findings from the present study are in support of lesion level dependent differences in global function. We observed no difference in global diastolic function 
TABLE 2 | Indices of LV structure and global systolic and diastolic function.

\begin{tabular}{|c|c|c|c|c|}
\hline Variable & TETRA & PARA & $P$-value & Cohen's $d$ \\
\hline \multicolumn{5}{|l|}{ STRUCTURE } \\
\hline $\begin{array}{l}\text { Aortic annulus } \\
\text { diameter (cm) }\end{array}$ & $2.26 \pm 0.13$ & $2.38 \pm 0.15$ & 0.118 & 0.85 \\
\hline LVID d (cm) & $4.86 \pm 0.40$ & $4.92 \pm 0.21$ & 0.341 & 0.19 \\
\hline SWT d (cm) & $0.91 \pm 0.06$ & $0.90 \pm 0.10$ & 0.869 & 0.12 \\
\hline PWT d (cm) & $0.84 \pm 0.05$ & $0.83 \pm 0.12$ & 0.956 & 0.11 \\
\hline LVID s (cm) & $3.24 \pm 0.22$ & $3.32 \pm 0.43$ & 0.635 & 0.23 \\
\hline SWT s (cm) & $1.27 \pm 0.05$ & $1.18 \pm 0.16$ & 0.177 & 0.76 \\
\hline PWT s (cm) & $1.20 \pm 0.10$ & $1.26 \pm 0.10$ & 0.215 & 0.60 \\
\hline RWT & $0.34 \pm 0.04$ & $0.34 \pm 0.05$ & 0.789 & 0.00 \\
\hline $\begin{array}{l}\text { LVM Index } \\
\left(\mathrm{g} \cdot \mathrm{m}^{-2}\right)\end{array}$ & $79.3 \pm 10.1$ & $75.8 \pm 9.4$ & 0.491 & 0.36 \\
\hline EDV (ml) & $106 \pm 9$ & $117 \pm 10$ & 0.036 & 1.16 \\
\hline ESV (ml) & $44 \pm 3$ & $46 \pm 11$ & 0.551 & 0.25 \\
\hline \multicolumn{5}{|c|}{ GLOBAL SYSTOLIC FUNCTION } \\
\hline SV (ml) & $62 \pm 9$ & $71 \pm 6$ & 0.016 & 1.18 \\
\hline $\begin{array}{l}\text { Heart rate } \\
\text { (bpm) }\end{array}$ & $57 \pm 11$ & $71 \pm 6$ & 0.008 & 1.58 \\
\hline$\dot{Q}(\llcorner/ \min )$ & $3.5 \pm 0.6$ & $5.0 \pm 0.9$ & 0.002 & 1.96 \\
\hline EF (\%) & $59 \pm 4$ & $61 \pm 7$ & 0.394 & 0.35 \\
\hline \multicolumn{5}{|c|}{ GLOBAL DIASTOLIC FUNCTION } \\
\hline$E(\mathrm{~cm} / \mathrm{s})$ & $86 \pm 12$ & $84 \pm 7$ & 0.733 & 0.20 \\
\hline $\mathrm{A}(\mathrm{cm} / \mathrm{s})$ & $43 \pm 6$ & $47 \pm 12$ & 0.373 & 0.42 \\
\hline$E / A$ & $2.02 \pm 0.18$ & $1.90 \pm 0.50$ & 0.544 & 0.32 \\
\hline IVRT (ms) & $81 \pm 6$ & $75 \pm 14$ & 0.326 & 0.56 \\
\hline
\end{tabular}

Data are mean $\pm S D$. A, late transmitral filling velocity; $d$, end-diastolic; $E$, early transmitral filling velocity; EDV, end-diastolic volume; EF, ejection fraction; ESV, end-systolic volume; IVRT, isovolumetric relaxation time; LVID, left ventricular internal diameter; LVM, left ventricular mass; PWT, posterior wall thickness $\dot{Q}$, cardiac output; RWT, relative wall thickness; s, end-systolic; SV, stroke volume; SWT, septal wall thickness. Bolded values in P-value column indicate significant between-group differences.

between TETRA and PARA, while SV and $\dot{Q}$ were lower in TETRA compared to PARA. The reduction in SV and subsequently $\dot{Q}$ are likely attributed to a smaller EDV given ESV was similar between groups, which implies reduced cardiac filling was likely responsible for the attenuated SV. LV dimensions were similar between groups; therefore the observed reduction in EDV is unlikely attributed to a smaller ventricle. While no investigations have directly compared LV dimensions between TETRA and PARA athletes, Kessler et al. (1986) observed smaller dimensions in sedentary individuals with tetraplegia compared to sedentary individuals with paraplegia. A more recent investigation using a pooled sample of athletes with tetraplegia and paraplegia observed no difference in LV dimensions when compared to able-bodied individuals and larger dimensions when compared to sedentary individuals with tetraplegia and paraplegia (De Rossi et al., 2014). Therefore, our observation of similar dimensions between the two groups is not unusual, and is likely attributed to the training status of our sample.

Contrary to the global systolic indices, systolic mechanics appear to be higher in TETRA relative to PARA. This includes
TABLE 3 | Peak systolic and diastolic LV mechanics.

\begin{tabular}{|c|c|c|c|c|}
\hline Variable & TETRA & PARA & $P$-value & Cohen's d \\
\hline \multicolumn{5}{|l|}{ PEAK (SYSTOLE) } \\
\hline Basal Rot (degrees) & $-7.3 \pm 2.2$ & $-4.4 \pm 1.1$ & 0.005 & 1.67 \\
\hline Apical Rot (degrees) & $13.4 \pm 3.3$ & $14.4 \pm 9.2$ & 0.791 & 0.14 \\
\hline Twist (degrees) & $19.5 \pm 3.4$ & $16.7 \pm 10.3$ & 0.527 & 0.37 \\
\hline$\varepsilon_{\mid}(\%)$ & $-18 \pm 3$ & $-17 \pm 2$ & 0.344 & 0.39 \\
\hline \multicolumn{5}{|l|}{$\varepsilon_{r}(\%)$} \\
\hline Basal Level & $34 \pm 11$ & $30 \pm 19$ & 0.568 & 0.26 \\
\hline Mid Level & $34 \pm 16$ & $32 \pm 11$ & 0.774 & 0.15 \\
\hline Apical Level & $33 \pm 17$ & $13 \pm 7$ & 0.060 & 1.54 \\
\hline \multicolumn{5}{|l|}{$\varepsilon_{C}(\%)$} \\
\hline Basal Level & $-19 \pm 7$ & $-14 \pm 5$ & 0.147 & 0.82 \\
\hline Mid Level & $-23 \pm 4$ & $-15 \pm 6$ & 0.010 & 1.57 \\
\hline Apical Level & $-36 \pm 10$ & $-23 \pm 5$ & 0.010 & 1.64 \\
\hline $\begin{array}{l}\text { Basal RotR } \\
\text { (degrees.s }^{-1} \text { ) }\end{array}$ & $-84 \pm 26$ & $-59 \pm 21$ & 0.047 & 1.06 \\
\hline 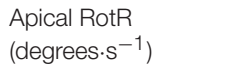 & $91 \pm 30$ & $89 \pm 28$ & 0.874 & 0.07 \\
\hline $\begin{array}{l}\text { Twist velocity } \\
\text { (degrees.s }^{-1} \text { ) }\end{array}$ & $129 \pm 26$ & $110 \pm 31$ & 0.218 & 0.66 \\
\hline \multicolumn{5}{|l|}{$\operatorname{SR}\left(s^{-1}\right)$} \\
\hline $\mathrm{SR}_{1}$ & $-0.92 \pm 0.20$ & $-0.91 \pm 0.18$ & 0.904 & 0.05 \\
\hline SRr basal & $1.90 \pm 0.35$ & $1.87 \pm 1.0$ & 0.946 & 0.04 \\
\hline $\mathrm{SR}_{\mathrm{r}}$ mid & $1.40 \pm 0.61$ & $1.69 \pm 0.34$ & 0.268 & 0.59 \\
\hline $\mathrm{SR}_{\mathrm{r}}$ apical & $1.71 \pm 0.76$ & $1.06 \pm 0.51$ & 0.173 & 1.00 \\
\hline $\mathrm{SR}_{\mathrm{C}}$ basal & $-1.25 \pm 0.34$ & $-0.93 \pm 0.35$ & 0.083 & 0.93 \\
\hline $\mathrm{SR}_{\mathrm{c}}$ mid & $-1.27 \pm 0.17$ & $-1.04 \pm 0.38$ & 0.137 & 0.78 \\
\hline $\mathrm{SR}_{\mathrm{c}}$ apical & $-2.40 \pm 1.13$ & $-1.85 \pm 0.25$ & 0.487 & 0.67 \\
\hline \multicolumn{5}{|l|}{ PEAK (DIASTOLE) } \\
\hline $\begin{array}{l}\text { Basal RotR } \\
\text { (degrees.s }^{-1} \text { ) }\end{array}$ & $55 \pm 18$ & $33 \pm 12$ & 0.013 & 1.44 \\
\hline 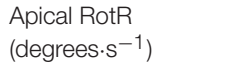 & $-78 \pm 34$ & $-85 \pm 42$ & 0.751 & 0.18 \\
\hline $\begin{array}{l}\text { Untwisting velocity } \\
\left.\text { (degrees. } s^{-1}\right)\end{array}$ & $-107 \pm 22$ & $-84 \pm 30$ & 0.110 & 0.87 \\
\hline \multicolumn{5}{|l|}{$\operatorname{SR}\left(s^{-1}\right)$} \\
\hline $\mathrm{SR}_{1}$ & $1.32 \pm 0.32$ & $1.15 \pm 0.16$ & 0.246 & 0.67 \\
\hline $\mathrm{SR}_{r}$ basal & $-1.64 \pm 0.56$ & $-2.26 \pm 1.38$ & 0.273 & 0.59 \\
\hline $\mathrm{SR}_{r}$ mid & $-2.00 \pm 0.80$ & $-2.44 \pm 1.38$ & 0.455 & 0.39 \\
\hline $\mathrm{SR}_{\mathrm{r}}$ apical & $-1.97 \pm 1.19$ & $-2.16 \pm 1.61$ & 0.840 & 0.13 \\
\hline $\mathrm{SR}_{\mathrm{C}}$ basal & $1.29 \pm 0.32$ & $1.31 \pm 0.51$ & 0.931 & 0.05 \\
\hline $\mathrm{SR}_{\mathrm{C}}$ mid & $1.90 \pm 0.63$ & $1.20 \pm 0.51$ & 0.028 & 1.22 \\
\hline $\mathrm{SR}_{\mathrm{C}}$ apical & $3.03 \pm 0.71$ & $1.99 \pm 0.69$ & 0.009 & 1.49 \\
\hline
\end{tabular}

Data are mean $\pm S D$. c, circumferential; $\varepsilon$, strain; l, longitudinal; r, radial; Rot, rotation; RotR, rotation rate; $S R$, strain rate. Bolded values in $P$-value column indicate significant between-group differences.

higher basal Rot and circumferential $\varepsilon$ at the level of the papillary muscle and apex, and a faster basal RotR. The $\varepsilon$-values reported in our TETRA sample are similar to values reported in the able-bodied literature (Yingchoncharoen et al., 2013). It is, therefore, unlikely that systolic mechanics are enhanced in the TETRA group, but rather the values in PARA group are lower. Head-down bed rest, which typically mirrors the 

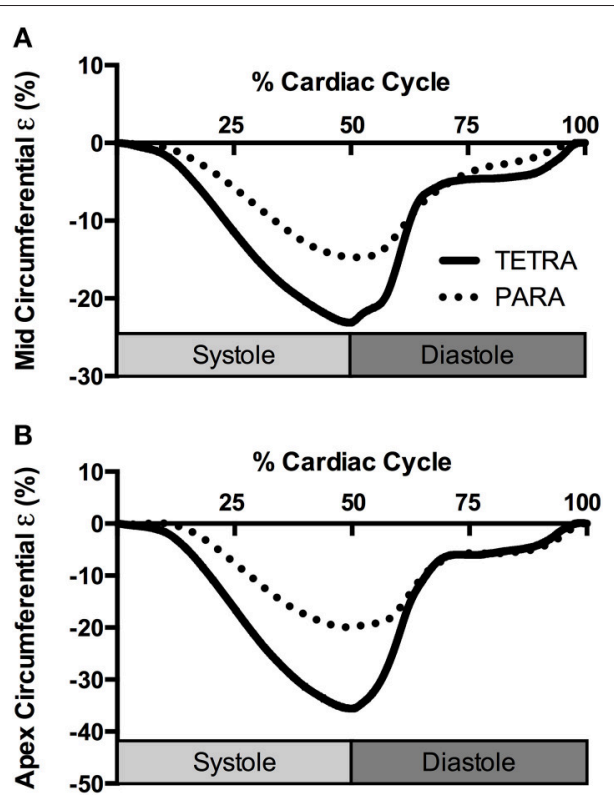

FIGURE 2 | Group averaged circumferential strain $(\varepsilon)$ traces at the level of the papillary muscle (A) and apex (B) for TETRA (solid line) and PARA (dotted line), normalized to percentage of the cardiac cycle.

cardiac adaptations following SCI, has been shown to elicit a reduction in SV, $\varepsilon$ and systolic SR in able-bodied individuals (Kozakova et al., 2011; Scott et al., 2014). The observation of preserved systolic mechanics and reduced SV in TETRA was therefore unanticipated, and may be attributed to their hemodynamic state. In particular, reductions in afterload have been shown to increase systolic mechanics (Burns et al., 2010); therefore the lower blood pressure in our TETRA group may have created an environment where systolic mechanics can be maintained despite reductions in loading. Hypotension has consistently been demonstrated in sedentary (West et al., 2012b) and athletic (West et al., 2014) individuals with tetraplegia, and is attributed to the disruption of descending sympathetic input to the vasculature.

The observation of lower systolic and diastolic mechanics in our PARA group may be indicative of two scenarios: LV dysfunction/disease or a "resetting" of resting mechanics. Lower resting LV mechanics have been documented in clinical populations (Leung and $\mathrm{Ng}, 2010$ ); however, we believe our finding is not pathological since global LV diastolic and systolic function (E/A and EF) were in a normal range (Lang et al., 2005; Dalen et al., 2010). The "resetting" of LV mechanics to lower resting values have been observed in high performance able-bodied athletes (Richand et al., 2007; Nottin et al., 2008), and while the mechanisms behind this adaptation are presently unknown, it is believed to create a large range for cardiovascular adjustments during exercise (Nottin et al., 2008). Both systolic and diastolic mechanics are increased during exercise in ablebodied individuals (Notomi et al., 2008; Stohr et al., 2011; Lee et al., 2012; Hensel et al., 2014). In addition to the obvious association between elevations in SV and $\varepsilon$, the augmentation of diastolic mechanics during exercise is key to promoting efficient diastolic filling, which in turn supports the elevations in SV (Notomi et al., 2008). It is presently unknown if exercise training in individuals with SCI elicits similar changes in LV mechanics as high-performance able-bodied athletes. Given cross-sectional evidence that athletes with paraplegia experience more favorable LV adaptations than athletes with tetraplegia (De Rossi et al., 2014), we postulate that involvement in highperformance sports and its associated large volume of exercise training may be capable of modifying LV mechanics to a lower resting value in PARA. Whether this modification has any impact on LV performance during exercise remains to be determined since LV performance per se has never been investigated during exercise post-SCI. Indeed, the few studies that have investigated the cardiac response to exercise in SCI have focused on measures of SV and report conflicting results, with some studies reporting increases in SV during armexercise (Davis et al., 1987; Hopman et al., 1992; Raymond et al., 1999), and others reporting no change (Davis and Shephard, 1988; Hopman et al., 1993, 1998; Raymond et al., 1999, 2001; Theisen et al., 2001). The discrepancy in these observations may be attributed to differences in the method used to estimate SV (ex. $\mathrm{CO}_{2}$ rebreathing, echocardiography, or impedance cardiography) and the acute exercise protocol (submaximal vs. maximal arm exercise). Nevertheless, the absence of apparent LV mechanical dysfunction at rest in high-performance athletes with SCI in the present study suggests any abnormal SV responses during exercise are likely attributable to reduced loading.

\section{LIMITATIONS}

The absence of an able-bodied control group makes it difficult to draw conclusions regarding the direction of the LV mechanics. While global LV outcomes suggest the PARA group is free from LV dysfunction, it is possible that the attenuated LV mechanics values are indicative of impending cardiac dysfunction. Conversely, the TETRA group may have experienced an "enhancement" of LV mechanics to accommodate the cardiovascular requirements of their endurance sport. In order to fully understand the influence of lesion level of LV mechanics in Paralympic athletes, future research should attempt to evaluate athletes in similar sporting events, especially sports in which TETRA and PARA athletes are in competition against each other (ex. wheelchair basketball or Para-cycling team relays).

\section{CONCLUSION}

In conclusion, high-performance athletes with TETRA and PARA experience differential changes in LV indices. While we would speculate these changes are attributed to lesion level, they may also be influenced by their training modality. TETRA athletes can be characterized as having normal diastolic function but reduced systolic function, an observation likely attributed to impaired venous return. On the other hand, PARA athletes demonstrated lower values for LV mechanics yet had 
no evidence of global systolic or diastolic dysfunction. We speculate this observation may be attributed to training induced improvements in LV efficiency rather than indicative of inherent LV dysfunction.

\section{AUTHOR CONTRIBUTIONS}

KC-Conception of the work; data acquisition, analysis and interpretation; drafting and revising manuscript; approved final copy; and agrees to be accountable. CW-Conception of the work; data acquisition and interpretation; critically revised manuscript; approved final copy; and agrees to be accountable. AK-Conception of the work; data acquisition and interpretation; revising manuscript; approved final copy; and agrees to be accountable.

\section{REFERENCES}

Akagawa, E., Murata, K., Tanaka, N., Yamada, H., Miura, T., Kunichika, H., et al. (2007). Augmentation of left ventricular apical endocardial rotation with inotropic stimulation contributes to increased left ventricular torsion and radial strain in normal subjects: quantitative assessment utilizing a novel automated tissue tracking technique. Circ. J. 71, 661-668. doi: 10.1253/circj.71.661

Burns, A. T., La Gerche, A., D’hooge, J., Macisaac, A. I., and Prior, D. L. (2010). Left ventricular strain and strain rate: characterization of the effect of load in human subjects. Eur. J. Echocardiogr. 11, 283-289. doi: 10.1093/ejechocard/jep214

Claydon, V. E., Hol, A. T., Eng, J. J., and Krassioukov, A. V. (2006). Cardiovascular responses and postexercise hypotension after arm cycling exercise in subjects with spinal cord injury. Arch. Phys. Med. Rehabil. 87, 1106-1114. doi: 10.1016/j.apmr.2006.05.011

Currie, K. D., West, C. R., Hubli, M., Gee, C. M., and Krassioukov, A. V. (2015). Peak heart rates and sympathetic function in tetraplegic nonathletes and athletes. Med. Sci. Sports Exerc. 47, 1259-1264. doi: 10.1249/MSS.0000000000000514

Dalen, H., Thorstensen, A., Vatten, L. J., Aase, S. A., and Stoylen, A. (2010). Reference values and distribution of conventional echocardiographic Doppler measures and longitudinal tissue Doppler velocities in a population free from cardiovascular disease. Circ. Cardiovasc. Imaging 3, 614-622. doi: 10.1161/CIRCIMAGING.109.926022

Davis, G. M., and Shephard, R. J. (1988). Cardiorespiratory fitness in highly active versus inactive paraplegics. Med. Sci. Sports Exerc. 20, 463-468. doi: 10.1249/00005768-198810000-00006

Davis, G. M., Shephard, R. J., and Leenen, F. H. (1987). Cardiac effects of short term arm crank training in paraplegics: echocardiographic evidence. Eur. J. Appl. Physiol. Occup. Physiol. 56, 90-96. doi: 10.1007/BF00696382

De Rossi, G., Matos-Souza, J. R., Costa, E. S., Campos, L. F., Santos, L. G., et al. (2014). Physical activity and improved diastolic function in spinal cord-injured subjects. Med. Sci. Sports Exerc. 46, 887-892. doi: 10.1249/MSS.0000000000000187

Dela, F., Mohr, T., Jensen, C. M., Haahr, H. L., Secher, N. H., BieringSørensen, F., et al. (2003). Cardiovascular control during exercise: insights from spinal cord-injured humans. Circulation 107, 2127-2133. doi: 10.1161/01.CIR.0000065225.18093.E4

Devereux, R. B., Alonso, D. R., Lutas, E. M., Gottlieb, G. J., Campo, E., Sachs, I., et al. (1986). Echocardiographic assessment of left ventricular hypertrophy: comparison to necropsy findings. Am. J. Cardiol. 57, 450-458. doi: 10.1016/0002-9149(86)90771-X

Du Bois, D., and Du Bois, E. F. (1989). A formula to estimate the approximate surface area if height and weight be known. 1916. Nutrition 5, 303-311; discussion 312-303.

Gates, P. E., Campbell, I. G., and George, K. P. (2002). Absence of training-specific cardiac adaptation in paraplegic athletes. Med. Sci. Sports Exerc. 34, 1699-1704. doi: 10.1097/00005768-200211000-00002

\section{FUNDING}

Research at the Paralympic events was supported by donations from the International Collaboration on Repair Discoveries, Coloplast, Wellspect HealthCare, and the Shkreli Foundation. Dr. KC was supported by a Craig H. Neilsen Foundation Postdoctoral Fellowship (281863).

\section{ACKNOWLEDGMENTS}

The authors would like to thank the athletes for taking the time to participate in our study, and GE Healthcare for generously lending us an ultrasound during field-testing. We would also like to acknowledge the International Paralympics Committee for providing us the opportunity to perform field-based assessments of Paralympic athletes.

Hasselberg, N. E., Haugaa, K. H., Sarvari, S. I., Gullestad, L., Andreassen, A. K., Smiseth, O. A., et al. (2015). Left ventricular global longitudinal strain is associated with exercise capacity in failing hearts with preserved and reduced ejection fraction. Eur. Heart J. Cardiovasc. Imaging 16, 217-224. doi: 10.1093/ehjci/jeu277

Hensel, K. O., Jenke, A., and Leischik, R. (2014). Speckle-tracking and tissueDoppler stress echocardiography in arterial hypertension: a sensitive tool for detection of subclinical LV impairment. Biomed Res. Int. 2014, 472562. doi: $10.1155 / 2014 / 472562$

Hopman, M. T., Monroe, M., Dueck, C., Phillips, W. T., and Skinner, J. S. (1998). Blood redistribution and circulatory responses to submaximal arm exercise in persons with spinal cord injury. Scand. J. Rehabil. Med. 30, 167-174. doi: 10.1080/003655098444101

Hopman, M. T., Oeseburg, B., and Binkhorst, R. A. (1992). Cardiovascular responses in paraplegic subjects during arm exercise. Eur. J. Appl. Physiol. Occup. Physiol. 65, 73-78. doi: 10.1007/BF01466277

Hopman, M. T., Pistorius, M., Kamerbeek, I. C., and Binkhorst, R. A. (1993). Cardiac output in paraplegic subjects at high exercise intensities. Eur. J. Appl. Physiol. Occup. Physiol. 66, 531-535. doi: 10.1007/BF00634305

Hostettler, S., Leuthold, L., Brechbuhl, J., Mueller, G., Illi, S. K., and Spengler, C. M. (2012). Maximal cardiac output during arm exercise in the sitting position after cervical spinal cord injury. J. Rehabil. Med. 44, 131-136. doi: 10.2340/16501977-0913

Huonker, M., Schmid, A., Sorichter, S., Schmidt-Trucksab, A., Mrosek, P., and Keul, J. (1998). Cardiovascular differences between sedentary and wheelchairtrained subjects with paraplegia. Med. Sci. Sports Exerc. 30, 609-613. doi: 10.1097/00005768-199804000-00020

Jacobs, P. L. (2009). Effects of resistance and endurance training in persons with paraplegia. Med. Sci. Sports Exerc. 41, 992-997. doi: 10.1249/MSS.0b013e318191757f

Jacobs, P. L., Mahoney, E. T., Robbins, A., and Nash, M. (2002). Hypokinetic circulation in persons with paraplegia. Med. Sci. Sports Exerc. 34, 1401-1407. doi: 10.1097/00005768-200209000-00001

Kessler, K. M., Pina, I., Green, B., Burnett, B., Laighold, M., Bilsker, M., et al. (1986). Cardiovascular findings in quadriplegic and paraplegic patients and in normal subjects. Am. J. Cardiol. 58, 525-530. doi: 10.1016/0002-9149(86) 90027-5

Kirshblum, S. C., Burns, S. P., Biering-Sorensen, F., Donovan, W., Graves, D. E., Jha, A., et al. (2011). International standards for neurological classification of spinal cord injury (revised 2011). J. Spinal Cord Med. 34, 535-546. doi: 10.1179/204577211X13207446293695

Kozakova, M., Malshi, E., Morizzo, C., Pedri, S., Santini, F., Biolo, G., et al. (2011). Impact of prolonged cardiac unloading on left ventricular mass and longitudinal myocardial performance: an experimental bed rest study in humans. J. Hypertens. 29, 137-143. doi: 10.1097/HJH.0b013e32833f5e 01

Krassioukov, A. (2009). Autonomic function following cervical spinal cord injury. Respir. Physiol. Neurobiol. 169, 157-164. doi: 10.1016/j.resp.2009.08.003 
Lang, R. M., Bierig, M., Devereux, R. B., Flachskampf, F. A., Foster, E., Pellikka, P. A., et al. (2005). Recommendations for chamber quantification: a report from the American Society of echocardiography's guidelines and standards committee and the chamber quantification writing group, developed in conjunction with the European association of echocardiography, a branch of the European society of cardiology. J. Am. Soc. Echocardiogr. 18, 1440-1463. doi: 10.1016/j.echo.2005.10.005

Lee, L. S., Mariani, J. A., Sasson, Z., and Goodman, J. M. (2012). Exercise with a twist: left ventricular twist and recoil in healthy young and middle-aged men, and middle-aged endurance-trained men. J. Am. Soc. Echocardiogr. 25, 986-993. doi: 10.1016/j.echo.2012.05.018

Leung, D. Y., and Ng, A. C. (2010). Emerging clinical role of strain imaging in echocardiography. Heart Lung Circ. 19, 161-174. doi: 10.1016/j.hlc.2009.11.006

Ma, C., Chen, J., Yang, J., Tang, L., Chen, X., Li, N., et al. (2014). Quantitative assessment of left ventricular function by 3-dimensional speckle-tracking echocardiography in patients with chronic heart failure: a meta-analysis. J. Ultrasound Med. 33, 287-295. doi: 10.7863/ultra.33.2.287

Maggioni, M. A., Ferratini, M., Pezzano, A., Heyman, J. E., Agnello, L., Veicsteinas, A., et al. (2012). Heart adaptations to long-term aerobic training in paraplegic subjects: an echocardiographic study. Spinal Cord 50, 538-542. doi: $10.1038 /$ sc.2011.189

Mor-Avi, V., Lang, R. M., Badano, L. P., Belohlavek, M., Cardim, N. M., Derumeaux, G., et al. (2011). Current and evolving echocardiographic techniques for the quantitative evaluation of cardiac mechanics: ASE/EAE consensus statement on methodology and indications endorsed by the Japanese Society of Echocardiography. Eur. J. Echocardiogr. 12, 167-205. doi: 10.1093/ejechocard/jer021

Notomi, Y., Popovic, Z. B., Yamada, H., Wallick, D. W., Martin, M. G., Oryszak, S. J., et al. (2008). Ventricular untwisting: a temporal link between left ventricular relaxation and suction. Am. J. Physiol. Heart Circ. Physiol. 294, H505-H513. doi: 10.1152/ajpheart.00975.2007

Nottin, S., Doucende, G., Schuster-Beck, I., Dauzat, M., and Obert, P. (2008). Alteration in left ventricular normal and shear strains evaluated by 2Dstrain echocardiography in the athlete's heart. J. Physiol. 586, 4721-4733. doi: 10.1113/jphysiol.2008.156323

Quiñones, M. A., Otto, C. M., Stoddard, M., Waggoner, A., Zoghbi, W. A., Doppler Quantification Task Force of The, Nomenclature and Standards Committee of the American Society of Echocardiography. et al. (2002). Recommendations for quantification of Doppler echocardiography: a report from the Doppler quantification task force of the nomenclature and standards committee of the American Society of echocardiography. J. Am. Soc. Echocardiogr. 15, 167-184. doi: $10.1067 / \mathrm{mje} .2002 .120202$

Raymond, J., Davis, G. M., Clarke, J., and Bryant, G. (2001). Cardiovascular responses during arm exercise and orthostatic challenge in individuals with paraplegia. Eur. J. Appl. Physiol. 85, 89-95. doi: 10.1007/s004210100449

Raymond, J., Davis, G. M., Climstein, M., and Sutton, J. R. (1999). Cardiorespiratory responses to arm cranking and electrical stimulation leg cycling in people with paraplegia. Med. Sci. Sports Exerc. 31, 822-828. doi: 10.1097/00005768-199906000-00010

Richand, V., Lafitte, S., Reant, P., Serri, K., Lafitte, M., Brette, S., et al. (2007). An ultrasound speckle tracking (two-dimensional strain) analysis of myocardial deformation in professional soccer players compared with healthy subjects and hypertrophic cardiomyopathy. Am. J. Cardiol. 100, 128-132. doi: 10.1016/j.amjcard.2007.02.063

Schmid, A., Huonker, M., Barturen, J. M., Stahl, F., Schmidt-Trucksass, A., Konig, D., et al. (1998a). Catecholamines, heart rate, and oxygen uptake during exercise in persons with spinal cord injury. J Appl Physiol 85, 635-641.

Schmid, A., Huonker, M., Stahl, F., Barturen, J. M., Konig, D., Heim, M., et al. (1998b). Free plasma catecholamines in spinal cord injured persons with different injury levels at rest and during exercise. J. Auton. Nerv. Syst. 68, 96-100. doi: 10.1016/S0165-1838(97)00127-6
Schumacher, Y. O., Ruthardt, S., Schmidt, M., Ahlgrim, C., Roecker, K., and Pottgiesser, T. (2009). Total haemoglobin mass but not cardiac volume adapts to long-term endurance exercise in highly trained spinal cord injured athletes. Eur. J. Appl. Physiol. 105, 779-785. doi: 10.1007/s00421-0080963-8

Scott, J., Downs, M., and Ploutz-Snyder, L. (2014). Temporal changes in left ventricular mechanics: impact of bed rest and exercise [Abstract]. J. Fed. Am. Soc. Exp. Biol. 28, abstract no. 884.23.

Spence, A. L., Naylor, L. H., Carter, H. H., Buck, C. L., Dembo, L., Murray, C. P., et al. (2011). A prospective randomised longitudinal MRI study of left ventricular adaptation to endurance and resistance exercise training in humans. J. Physiol. 589, 5443-5452. doi: 10.1113/jphysiol.2011.217125

Stohr, E. J., Gonzalez-Alonso, J., and Shave, R. (2011). Left ventricular mechanical limitations to stroke volume in healthy humans during incremental exercise. Am. J. Physiol. Heart Circ. Physiol. 301, H478-H487. doi: 10.1152/ajpheart.00314.2011

Theisen, D. (2012). Cardiovascular determinants of exercise capacity in the Paralympic athlete with spinal cord injury. Exp. Physiol. 97, 319-324. doi: 10.1113/expphysiol.2011.063016

Theisen, D., Vanlandewijck, Y., Sturbois, X., and Francaux, M. (2001). Central and peripheral haemodynamics in individuals with paraplegia during light and heavy exercise. J. Rehabil. Med. 33, 16-20. doi: 10.1080/1650197013000 06489

Thijssen, D. H., Steendijk, S., and Hopman, M. T. (2009). Blood redistribution during exercise in subjects with spinal cord injury and controls. Med. Sci. Sports Exerc. 41, 1249-1254. doi: 10.1249/MSS.0b013e31819 $6 \mathrm{c} 902$

Voigt, J. U., Pedrizzetti, G., Lysyansky, P., Marwick, T. H., Houle, H., Baumann, R., et al. (2015). Definitions for a common standard for 2D speckle tracking echocardiography: consensus document of the EACVI/ASE/Industry Task Force to standardize deformation imaging. Eur. Heart J. Cardiovasc. Imaging 16, 1-11. doi: 10.1093/ehjci/jeu184

Weidemann, F., Jamal, F., Kowalski, M., Kukulski, T., D’hooge, J., Bijnens, B., et al. (2002). Can strain rate and strain quantify changes in regional systolic function during dobutamine infusion, B-blockade, and atrial pacing-implications for quantitative stress echocardiography. J. Am. Soc. Echocardiogr. 15, 416-424. doi: $10.1067 / \mathrm{mje} .2002 .116535$

West, C. R., Campbell, I. G., Shave, R. E., and Romer, L. M. (2012a). Resting cardiopulmonary function in Paralympic athletes with cervical spinal cord injury. Med. Sci. Sports Exerc. 44, 323-329. doi: 10.1249/MSS.0b013e31822b7441

West, C. R., Mills, P., and Krassioukov, A. V. (2012b). Influence of the neurological level of spinal cord injury on cardiovascular outcomes in humans: a metaanalysis. Spinal Cord 50, 484-492. doi: 10.1038/sc.2012.17

West, C. R., Wong, S. C., and Krassioukov, A. V. (2014). Autonomic cardiovascular control in Paralympic athletes with spinal cord injury. Med. Sci. Sports Exerc. 46, 60-68. doi: 10.1249/MSS.0b013e31829e46f3

Yingchoncharoen, T., Agarwal, S., Popovic, Z. B., and Marwick, T. H. (2013). Normal ranges of left ventricular strain: a meta-analysis. J. Am. Soc Echocardiogr. 26, 185-191. doi: 10.1016/j.echo.2012.10.008

Conflict of Interest Statement: The authors declare that the research was conducted in the absence of any commercial or financial relationships that could be construed as a potential conflict of interest.

Copyright (c) 2016 Currie, West and Krassioukov. This is an open-access article distributed under the terms of the Creative Commons Attribution License (CC BY). The use, distribution or reproduction in other forums is permitted, provided the original author(s) or licensor are credited and that the original publication in this journal is cited, in accordance with accepted academic practice. No use, distribution or reproduction is permitted which does not comply with these terms. 